\title{
The COVID-19 testing debacle
}

\author{
Insufficient SARS-CoV-2 testing has left many nations flying blind as they reopen their economies. Without \\ adequate testing, new infections could outrun control measures.
}

C OVID-19 has laid bare the weaknesses of clinical diagnostics infrastructures. Underfunded, undervalued and underprepared, laboratory testing has been overwhelmed by the contagion. A combination of low reimbursement, low margins, low capacity and low investment has stifled innovation and competition. Cost-cutting, centralization and regulatory rigidity have created backlogs and slowed the tracking of infections. Given the pivotal role of diagnostics in healthcare decision-making, COVID-19 shows just how much clinical testing needs a radical rethink.

On May 28, the United States achieved a record 416,664 tests for SARS-CoV-2 in one day (around one test per thousand people per day). The United Kingdom, France, Spain and much of the rest of Europe are testing at a similar level. Given that only $\sim 3,000$ tests are carried out per day during a typical flu season, we are approaching the limit of testing capacity using existing clinical laboratories and supply chains. But it is nowhere near the level needed for countries to contain COVID-19 while reopening their economies.

Testing capacity determines how many cases a country can confirm. Insufficient testing underreports cases and deaths. More importantly, as economies reopen, insufficient testing relinquishes control of COVID-19 because new virus clusters elude detection and spark new outbreaks.

According to the World Health Organization, if more than $10 \%$ of results come back positive, testing capacity is insufficient; test positivity higher than $10 \%$ means many active infections are being missed. Nobel laureate economist Paul Romer has suggested 30 million daily tests are needed to reach the $10 \%$ positivity threshold in the United States. The Rockefeller Foundation Testing Action Plan projects 5 million tests by early June, growing to 20 million in the following four months. Even the lowest estimates suggest 750,000 to 900,000 molecular tests will be needed every day - more than double what is presently available.

The massive scale of testing now needed is a result of government failure at the beginning of the outbreak to quickly implement testing, tracking and tracing measures - together with social distancing - to limit the community spread of SARS-CoV-2. In those countries where lockdown was decisive and stringent tracking and tracing procedures were put in place - Taiwan, South Korea, Hong Kong and Singapore - subsequent massive testing has been unnecessary.

Elsewhere, failed lockdowns and slipshod testing have gone hand in hand. In the United States, the Centers for Disease Control botched its test rollout and the Food and Drug Administration (FDA) dithered on Emergency Use Authorizations (EUAs), vacillating between oversight that was too stringent for RT-PCR testing and too lax for serological testing. The United Kingdom abandoned broad testing and contact tracing in March, then reversed its policy. France planned widespread testing at the end of March, then gave up on diagnostic testing when it became clear its clinical testing infrastructure couldn't deliver.

The shrinking number of public labs, along with their centralization, has meant results can take days if not weeks to come back - useless for tracking and tracing. France has only 45 testing labs; the United States just 97 public labs, with a commercial sector dominated by corporate giants like Quest Diagnostics and LabCorp. Germany stands apart, with a robust infrastructure of 20 national reference centers, around 40 'consultant laboratories' and hundreds of hospital- and clinic-based local testing centers. Results from the extended network are coordinated through a computer system adapted from Germany's Antibiotic Resistance Surveillance network; Heidelberg even has a 'corona taxi' program that transports medics in protective gear to check on patients in their homes.

In contrast, testing in the United States is a mess. With shambolic coordination at the national level, existing providers have become swamped, testing backlogs have lengthened and shortages of swabs, supplies and reagents have grown. As traditional labs failed to ramp up, several research centers and hubs have stepped in and retooled to provide semi-automated 'pop up' labs for COVID-19, working with regulatory authorities and partnering with clinically accredited labs to innovate around reagent shortages. One open-source effort recently won an FDA EUA for processing $>10,000$ samples per day using automated loop-mediated isothermal amplification, circumventing RT-PCR reagent shortages and aiming to provide results in 24 hours. Another idea is to use multiplexing schemes and next-generation sequencing to pool thousands of bar-coded COVID-19 samples and then triage down to confirmatory testing for the identification of individual positives - an approach that enabled Wuhan, China to test 6.5 million people in 9 days.

Whatever technology is adopted, with tests in such short supply, strategic deployment to hotspots will be important in combination with contact tracing: clinical centers, essential services, travel hubs, nursing homes, meat-packing plants and places of congregation (bars, nightclubs or religious gatherings) will remain priorities, with drive-thru testing provided as an adjunct.

During reopening, testing volume will most likely be built by employers. Symptom reporting and contact-tracing apps built on Apple and Google's API will be used to identify workers to prioritize for testing (Drexel University and Baylor College already offer such an app).

Ultimately, kits for doctors' offices and at-home testing will become available, some perhaps even using CRISPR technology, like those from Mammoth Biosciences or Sherlock Biosciences.

The failure of the clinical diagnostic sector to deal with the demands of a 21 st century pandemic is a wake-up call. The traditional players appear incapable of innovating around the problem probably because innovation is alien in an environment where reimbursement cuts by the 2014 Protecting Access to Medicare Act, erosion of intellectual property protection, and a lack of regulatory clarity has depressed profit margins and stunted investment.

But infectious disease is not going away. And in an increasingly interconnected, urbanized and globalized world, COVID-19 has shown that present lab testing systems are clearly unfit for purpose. A reinvigoration of new ideas and new technology is urgently needed to put diagnostics back at the forefront of twenty-first-century medicine and pandemic prevention.

Published online: 3 June 2020 https://doi.org/10.1038/s41587-020-0575-3 